\title{
UNA NUEVA ESPECIE DE HYPERBAENA (MENISPERMACEAE) DE VERACRUZ, MEXICO
}

\author{
Eva Perez Cueto y Gonzalo Castillo-Campos \\ Instituto Nacional de Investigaciones sobre Recursos \\ Bióticos. Apartado postal 63, 91000 Xalapa, \\ Veracruz, México
}

\begin{abstract}
RESUMEN
Se describe una nueva especie de Menispermaceae, Hyperbaena jalcomulcensis Pérez \& Castillo-Campos, del centro de Veracruz, México. Se trata de un árbol que crece en selva baja caducifolia y mediana subperennifolia. Se discute su distribución, habitat y afinidades.
\end{abstract}

\section{ABSTRACT}

Hyperbaena jalcomulcensis is described as a new species in the Menispermaceae. It is a tree dwelling tropical deciduous and semideciduous forests of central Veracruz. Its distribution, habitat and affinities are discussed.

Recientes exploraciones y colecciones efectuadas en la región de Jalcomulco (Castillo, 1985 y Pérez, 1988) en el centro del estado de Veracruz, y la revisión de especímenes para la preparación del fascículo Menispermaceae de la Flora de Veracruz, han revelado la presencia de una nueva especie del género Hyperbaena Miers ex Bentham (Menispermaceae). Hyperbaena jalcomulcensis Pérez \& Castillo-Campos sp. nov. es semejante a Hyperbaena mexicana Miers y por lo tanto había sido confundida con el nombre de esta última especie. Hyperbaena jalcomulcensis sin embargo, se diferencia por presentar inflorescencia espigada con flores sésiles, mientras que $H$. mexicana tiene inflorescencia paniculada con flores pediceladas.

El género Hyperbaena consta de 19 especie nativas de América tropical, distribuídas desde el centro y sur de México e Islas del Caribe, hasta el norte de Argentina y Bolivia (Miers, 1867; Mathias y Theobald, 1981 y Pérez, 1988).

Hyperbaena jalcomulcensis Pérez \& Castillo-Campos, sp. nov.

Folia nervis basalibus 2, ex petiolo oriundibus, ad nervum medium parallelis usque ad tertium folii ascendentibus, nervis lateralibus 5-10 paribus. Inflorescentia masculina spicata; pedunculis 1-2 per axillam. Affinis $H$. mexicana (Fig. 1).

Arboles caducifolios, hasta de $20 \mathrm{~m}$ de altura, el tallo erecto, cilíndrico, glabro. Hojas alternas, simples, pecioladas; lámina elíptico-oblonga, $5-20 \mathrm{~cm}$ de largo, $1.5-6 \mathrm{~cm}$ de ancho, coriácea, glabra, margen entero, algunas veces ondulado, ápice agudo mucronulado, algunas 


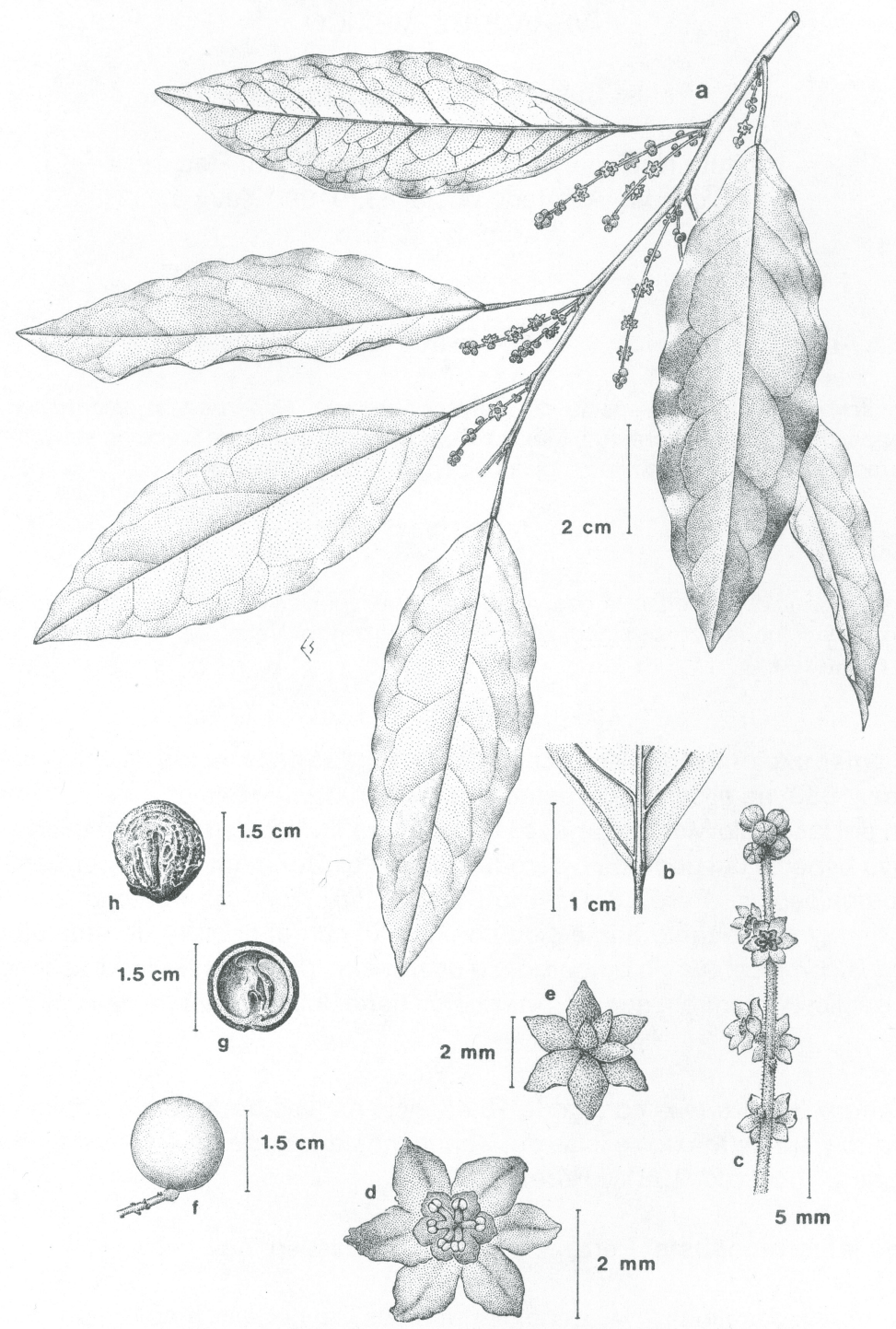

Figura 1. Hyperbaenajalcomulcensis. a. Rama con hojas e inflorescencias; b. Nervios basales de la hoja; c. Inflorescencia; d. Flor; $\Theta$. Sépalos de la flor; f. Fruto; g. Corte longitudinal del fruto; h. Semilla. Ilustración por Edmundo Saavedra, basada en los ejemplares Pérez Castillo \& Olivé 24; Acosta, Castillo \& Barradas 969; Castillo \& Medina 2958 y Robles 321. 
veces subcurvo, base obtusa a cuneada, haz verde-amarillento, envés verde pálido, la nervación anastomosada, broquidódroma finamente reticulada, nervios primarios con origen en el nervio medio, excepto 2 nervios basales con origen en el peciolo que ascienden paralelos al nervio medio hasta 1/3 del largo de la hoja, nervio medio prominente en ambas superficies, pares de nervios laterales 5-10, poco prominentes en el haz, visibles en el envés, peciolos ascendentes, cilíndricos, estriados, $1.8-3 \mathrm{~cm}$ de largo, $0.1 \mathrm{~cm}$ de ancho, ampliándose a $0.2 \mathrm{~cm}$ en la unión de la base de la hoja, glabro. Inflorescencia supra axilar, 1-2 espigada, 0.7-4 cm de largo, con 10-25 flores; pedúnculos cilíndricos, 0.7-3.5 cm de largo, seríceo-tomentosos a glabros; brácteas lanceoladas, menores de $1 \mathrm{~mm}$ de largo, seríceas; bracteola 1 por flor, triangular, 0.7-1 mm de largo, 0.4-0.5 mm de ancho, en la parte externa serícea, en la parte interna glabra; flor masculina actinomorfa, amarilla; sépalos 8 en 2 series, antipétalos, erectodivaricados, sépalos externos 2(3), lanceolados, 1-1.5 mm de largo, 0.4-0.8 mm de ancho, subcarnosos, en la superficie abaxial pilosiúsculos a glabriúsculos, la superficie adaxial glabra, margen entero, ápice obtuso, base redondeada, sépalos internos (5)6, elípticos a obovados, 1.7-2.2 $\mathrm{mm}$ de largo, 0.5-1.7 $\mathrm{mm}$ de ancho, subcarnosos, externamente pilosiúsculos, internamente glabros, margen entero, ápice agudo-obtuso, base redondeada; pétalos 5-6 en un verticilo, antipétalos, erecto-divaricados, rombiformes, $0.8-1.2 \mathrm{~mm}$ de largo, 0.5-0.8 $\mathrm{mm}$ de ancho, subcarnosos, glabros, margen entero, ápice agudo, base truncada; estambres (5)6, en un verticilo, fasciculados, antipétalos, amarillos, elíptico-oblongos, algunas veces obcónicos, 0.4-0.8 mm de largo, 0.2-0.4 mm de ancho, subcarnosos, glabros, margen entero, anteras dorsifijas, biloculares, dehiscencia longitudinal-lateral, color café oscuro, 0.2-0.4 mm de largo, 0.2-0.5 mm de ancho, glabras. Fruto una drupa, sésil a lo largo del pedúnculo, verdeamarillento, globoso, 1-2 cm de diámetro, glabro, exocarpo delgado, crustáceo, mesocarpo crustáceo, endocarpo óseo; semilla obovoide, en forma de herradura, algo comprimida en la base; embrión linear.

Es conveniente mencionar que no se incluyen observaciones de flores femeninas debido a que los especímenes no se encontraban en floración.

TIPO: México. Veracruz; Mpio. Jalcomulco, antes de llegar a Jalcomulco, $4 \mathrm{~km}$ después de la desviación, 700 m.s.n.m., 2 octubre de 1986, Pérez, Castillo \& Olivé 24 (Holotipo: XAL; isotipos, ENCB, F, MEXU, MO, NY).

Ejemplares adicionales examinados. México. Veracruz. Mpio. Actopan, Cañada de la Mesa al norte de Mozomboa, Acosta, Castillo \& Barradas 969 (XAL); Mpio. Axocuapan, Monte Rey, Ejido Coetzalan, Robles 321 (XAL, XALU); Ibid., Robles 335 (XAL, XALU); Mpio. Emiliano Zapata, Plan del Río, Hernández \& Dorantes 1827 (F, MEXU, XAL); Mpio. Jalcomulco, entre Jalcomulco y Apazapan, en la cañada que forma el río por el lado S, Castillo \& Golberg 1950 (XAL); Mpio. Jalcomulco, $3 \mathrm{~km}$ antes de llegar a Jalcomulco, en las faldas de la mesa de Barro Negro, Castillo \& Lorence 2807 (XAL); Mpio. Jalcomulco, $3 \mathrm{~km}$ antes de llegar a Jalcomulco en los cantiles de las faldas del cerro, Castillo \& Medina 2958 (XAL); Mpio. Jalcomulco, antes de llegar a Jalcomulco, 4 km después de la desviación, Pérez, Castillo \& Olivé 23 (XAL); Ibid., Pérez \& Olivé 41 (XAL); Mpio. Puente Nacional, Rinconada, Ventura 12295 (ENCB, MEXU).

Distribución. México. Veracruz. Conocida solamente para el centro del Estado (Fig. 2). 


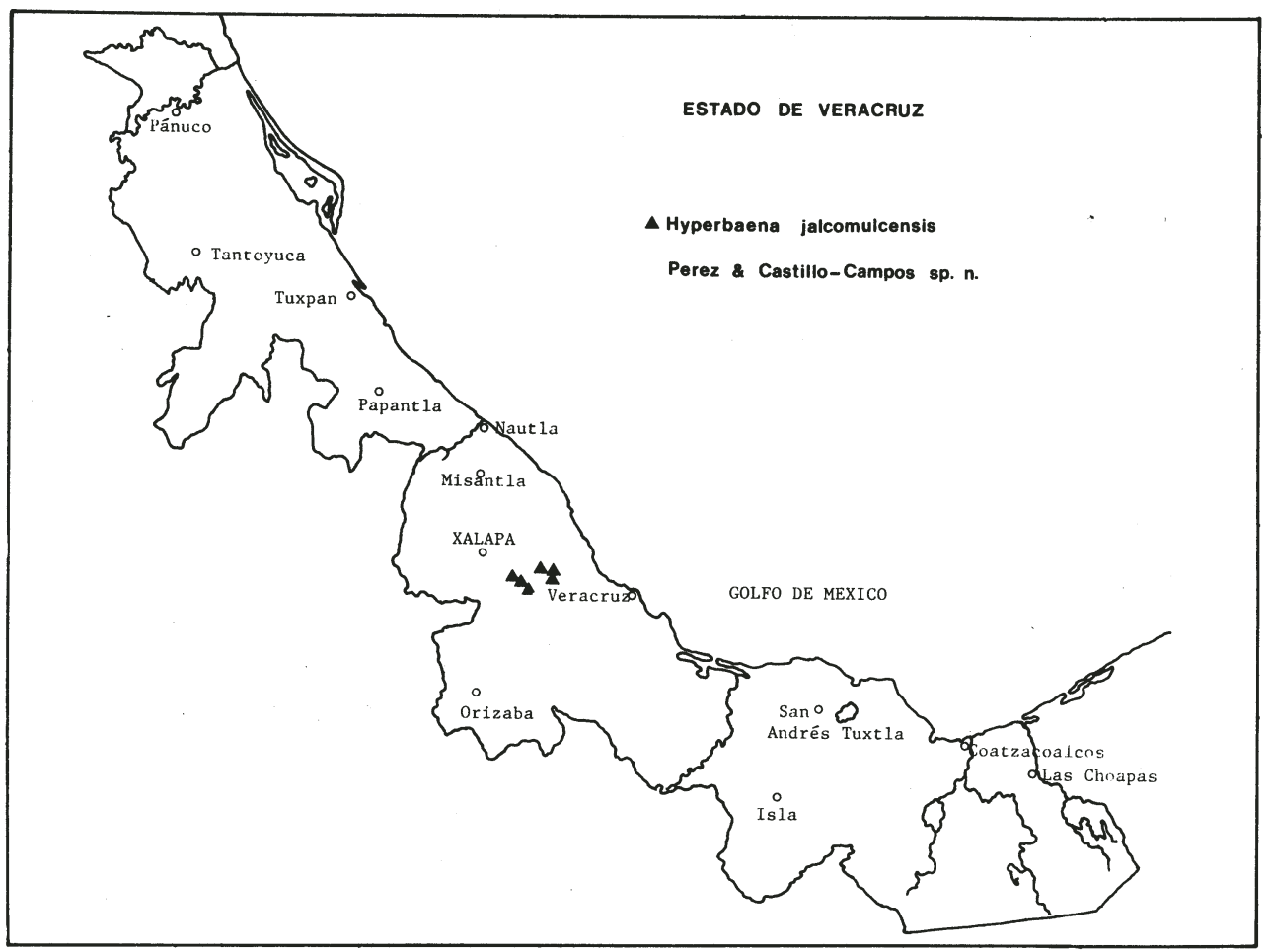

Figura 2. Mapa de distribución de Hyperbaena jalcomulcensis.

Habitat. Hyperbaena jalcomulcensis prospera en climas tropicales con temperaturas medias anuales cercanas a $25^{\circ} \mathrm{C}$ y una precipitación total anual de 800 a $1200 \mathrm{~mm}$, con una estación seca y una lluviosa bien marcadas. Los suelos son rendzinas y litosoles que se encuentran en sustrato de roca caliza. Se localiza principalmente en las faldas y fondos de las barrancas más húmedas, en la selva baja caducifolia y selva mediana subperennifolia, asociada con Brosimum alicastrum Sw., Bursera simaruba (L.) Sarg., Manilkara zapota (L.) Van Royen, Antirhea aromatica Castillo-Campos \& Lorence, Comocladia engleriana Loes., Ceiba aesculifolia (H.B.K.) Britton \& Baker, Bauhinia divaricata L., Ficus tecolutlensis (Liebm.) Miq. y Protium copal (Schldl. \& Cham.) Engl., entre los 150 y $700 \mathrm{~m}$ de altitud. Florece de enero a octubre.

Afinidades. Hyperbaena jalcomulcensis está estrechamente relacionada con Hyperbaena mexicana Miers, cuyas características diferenciales están dadas en el cuadro 1. 
Pérez y Castillo-Campos: Una Nueva Especie de Hyperbaena

Cuadro 1. Caracteres morfológicos diagnósticos de las dos especies de Hyperbaena conocidas para Veracruz

\begin{tabular}{|l|l|l|}
\hline Carácter & H. jalcomulcensis & H. mexicana \\
\hline Hoja & elíptico-oblonga & elíptica \\
\hline Nervios basales & $\begin{array}{l}\text { 2, paralelos al nervio medio } \\
\text { hasta 1/3 de la lámina }\end{array}$ & 2 arqueados \\
\hline Nervios laterales & $\begin{array}{l}5-10 \text { pares } \\
\text { Inflorescencia }\end{array}$ & espigada pares \\
\hline Flores & sésiles & paniculada \\
\hline $\begin{array}{l}\text { Número de inflorescen- } \\
\text { cias por axila }\end{array}$ & $\begin{array}{l}1-2 \\
\text { Pedúnculos }\end{array}$ & $\begin{array}{l}\text { seríceo-tomentosos } \\
\text { a glabros }\end{array}$ \\
\hline
\end{tabular}

\section{AGRADECIMIENTOS}

Los autores desean expresar su agradecimiento a Edmundo Saavedra por la elaboración de las figuras, al Dr. Victor Rico Gray, M. en C. Andrés Vovides, Biól. Rocío Jiménez y Biól. Héctor Narave por la revisión crítica del manuscrito, al C. Victor Olivé por el apoyo de campo y a Margarita Rebolledo por el mecanografiado del texto.

\section{LITERATURA CITADA}

Castillo, G. 1985. Integración de paisajes en la región de Jalcomulco, Veracruz. Tesis de Licenciatura. Facultad de Biologia. Universidad Veracruzana. Xalapa. 110 pp.

Mathias, M. E. \& W. L. Theobald. 1981. A revision of the genus Hyperbaena (Menispermaceae). Brittonia 33(1): 81-104.

Miers, J. 1867. Hyperbaena. On the Menispermaceae. Ann. Mus. Nat. Hist. III. 19: 84-95.

Pérez, E. 1988. Estudio taxonómico de la familia Menispermaceae en el estado de Veracruz. Tesis de Licenciatura. Facultad de Biología. Universidad Veracruzana. Xalapa. 85 pp. 